\title{
ORBITS OF THE PSEUDOCIRCLE
}

\author{
JUDY KENNEDY AND JAMES T. ROGERS, JR. ${ }^{1}$
}

ABSTRACT. The following theorem is proved.

THEOREM. The pseudocircle has uncountably many orbits under the action of its homeomorphism group. Each orbit is the union of uncountably many composants.

A pseudocircle is a circularly chainable, hereditarily indecomposable, separating plane continuum. R. H. Bing [1] constructed a pseudocircle in 1951 and asked two questions. Are any two pseudocircles homeomorphic? Is a pseudocircle homogeneous?

In 1968, L. Fearnley [6] proved that the answer to the first question is yes, and Fearnley [5] and J. T. Rogers, Jr. [14] independently proved that the answer to the second question is no. Since the advent of the Effros theorem, several elegant proofs of the nonhomogeneity of the pseudocircle have appeared $[\mathbf{7}, \mathbf{1 0}$, and 13].

In 1968, after seeing the proof that the pseudocircle is not homogeneous, $F$. B. Jones asked the second author how many orbits the pseudocircle had under the action of its homeomorphism group, but the matter was not pursued further. Recently, however, the question has arisen again, and Wayne Lewis [3] has asked if the pseudocircle has uncountably many orbits.

The purpose of this paper is to prove the following theorem, which gives an affirmative answer to this question.

THEOREM. The pseudocircle has uncountably many orbits under the action of its homeomorphism group. Each of these orbits is the union of uncountably many composants.

An interesting sidelight of the proof is the construction of two homeomorphic sets, one being an open set of the pseudocircle and the other being an open set of the pseudoarc.

During the proof we construct an uncountable, abelian subgroup of the homeomorphism group of the pseudocircle. The homeomorphism constructed by Handel [8] might be an element of this group.

Each orbit of the pseudocircle is a Borel set [4]. Lewis has announced that no orbit can be a $G_{\delta}$. In the course of our argument we prove this, but we do not determine further restrictions on the type of the Borel set for the orbit.

Question. What classes of Borel sets occur as the orbits of the pseudocircle? In particular, are each two orbits of the same class?

Received by the editors May 1, 1984 and, in revised form, August 13, 1985.

1980 Mathematics Subject Classification. Primary 54F20, 54F50.

Key words and phrases. Continuum, pseudoarc, pseudocircle, orbit, Borel set, nearly homogeneous, stable homeomorphism.

${ }^{1}$ The research of the second author was partially supported by NSF Grant MCS-8300569.

(C) 1986 American Mathematical Society $0002-9947 / 86 \$ 1.00+\$ .25$ per page 


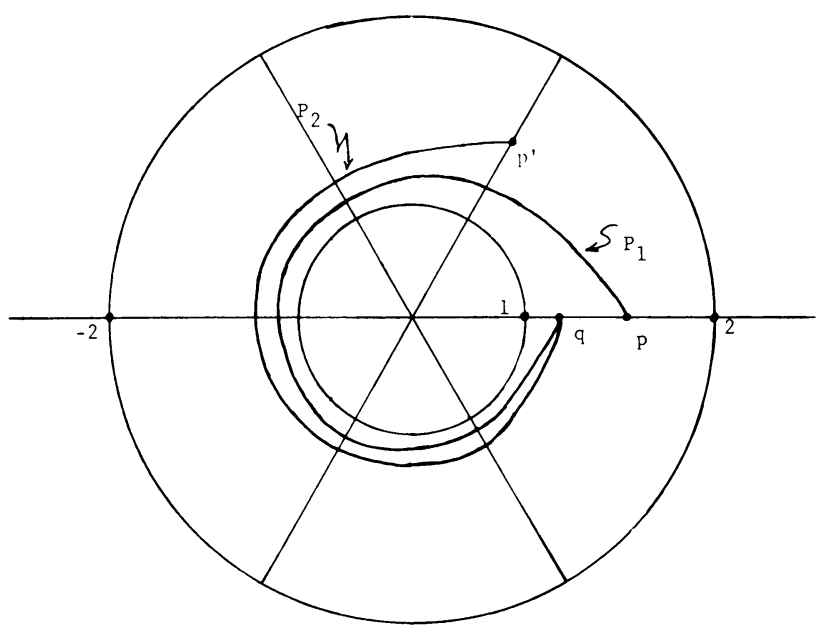

FIGURE 1

A continuum is circle-like or circularly chainable if it has covers of arbitrarily small mesh whose nerves are circles. An arc-like or chainable continuum is defined similarly.

A pseudoarc is an arc-like, hereditarily indecomposable continuum. Each proper nondegenerate subcontinuum of a pseudocircle is a pseudoarc. The pseudoarc has the fixed-point property $[8]$.

If $X$ is a compactum, then $H(X)$ denotes its homeomorphism group with the compact-open topolgy. The orbit of a point $x$ in $X$ is $\{y: h(y)=x$, for some $h$ in $H(X)\}$.

If $A$ is a collection of sets, then $A^{*}$ denotes the union of the sets in $A$. If $B$ is another collection of sets, then $B$ is an amalgamation of $A$ if $A^{*}=B^{*}$ and each set in $B$ is the union of some sets in $A$. If the closure of each set in $A$ is a subset of a set in $B$, then $A$ is said to closure-refine $B$. If $A$ is a chain cover of a continuum $X$, we shall assume each link of $A$ contains a point not in the closure of any other link of $A$. Such a chain is said to cover $X$ essentially.

1. Embedding an annulus in an annulus. The key idea of this section is to describe a wiggly embedding of an annulus into a standard annulus so that the embedded annulus is mapped onto itself by rotation through an angle of $\frac{\pi}{3}$. Afterwards, we hint at how to continue the process. Emphasis is on intuition; more rigor and detail is provided in the next section.

We use polar coordinates in the plane. We speak of chains with links being closed 2-cells that intersect only in an arc in their boundaries. One can get open sets as links by fattening these slightly.

Consider the closed, planar annulus $A$ bounded by the circles $r=1$ and $r=2$. Divide $A$ into 6 congruent parts invariant under rotation by $\pi / 3$, as indicated in Figure 1. Let $P_{1}$ be an arc in $A$ winding once around the origin in the counterclockwise direction, with initial point $p=\left(1 \frac{3}{4}, 0\right)$ and terminal point $q=\left(1 \frac{1}{4}, 0\right)$ and with $r$-coordinate decreasing monotonically. Let $P_{2}$ be a similar arc from $p^{\prime}=\left(1 \frac{3}{4}, \frac{\pi}{3}\right)$ to $q$. These arcs are pictured in Figure 1. 
Adjust $P_{1}$ and $P_{2}$ if necessary so that they intersect only in point $q$. Let $P=$ $P_{1} \cup P_{2}$. Let $h$ be the rotation of the plane by $\frac{\pi}{3}$. Note that $h(p)=p^{\prime}$. Adjust $P$ if necessary so that $P \cap h(P)=\left\{p^{\prime}\right\}$.

The union of the six $\operatorname{arcs} h^{n}(P), n=0,1, \ldots, 5$, is a simple closed curve $C_{2}$ in $A$ invariant under $h$. Thicken $C_{2}$ to a neighborhood $A_{2}$ homeomorphic to an annulus, so that $A_{2}$ is also invariant under $h$.

Let $B_{2}$ be the piece of $A_{2}$ that forms a thickening of $P$ so that the boundary of $B_{2}$ in $A_{2}$ consists of a short line segment $L_{1}$ intersecting $\theta=0$ and a line segment $L_{2}$ intersecting $\theta=\frac{\pi}{3}$ congruent to $L_{1}$ by the rotation of $\frac{\pi}{3}$. Divide $B_{2}$ with "radial" lines into links. Use $h$ to rotate $B_{2}$ around the annulus $A$ so that the links of $B_{2}$ determine $6 n$ links in $A_{2}$, where $n$ is some integer.

Consider a "rotation" of $A_{2}$ defined by moving each link homeomorphically onto its successor link. There are $6 n$ of these "rotations," including the identity. Note that the "rotations" corresponding to $0, n, 2 n, 3 n, 4 n$, and $5 n$ agree with rotations of the plane through multiples of $\frac{\pi}{3}$ (This is the heart of the construction.).

To continue, embed appropriately a simple closed curve $C_{3}$ in the annular neighborhood $A_{2}$ so that $C_{3}$ is invariant under the $6 n$ "rotations" of $A_{2}$. The curve $C_{3}$ will be the union of $6 n$ arcs, each beginning in some link of $A_{2}$ and ending in its successor link. Thicken $C_{3}$ to $A_{3}$, also invariant under these "rotations." Chop $A_{3}$ into links and continue.

2. An uncountable abelian group of homeomorphisms of the pseudocircle. The pseudocircle will be defined as the intersection of annuli, so we fill in some details and provide some rigor to the intuitive description of the previous section.

The first modification is that the $\operatorname{arcs} P_{1}$ and $P_{2}$ of the previous section must be embedded crookedly in $A$ with respect to the six links of $A$. Hence we lose the " $r$-coordinate decreasing monotonically." This was only used, however, as an aid to intuition. The $\operatorname{arcs} P_{1}, P_{2}$, and $P$ are still to enjoy all the properties of paragraph four of the previous section.

The second adjustment is that the simple closed curve $C_{2}$ is to be smoothly embedded. Choose the neighborhood $A_{2}$ of $C_{2}$ so that it is also smoothly embedded and thin.

Hence we have a smoothly embedded annulus $A_{2}$ and a smoothly embedded simple closed curve $C_{2}$ that are both invariant under the rotation $h$. The next step is to describe precisely the "links" and "rotations" of $A_{2}$.

Let $C_{2}^{\prime}$ be the circle in the plane centered at the origin such that the circumference of $C_{2}^{\prime}$ is equal to the length of $C_{2}$. Let $f_{2}: C_{2} \rightarrow C_{2}^{\prime}$ be a diffeomorphism that preserves length. Extend $f_{2}$ to a homeomorphism (also called $f_{2}$ ) taking $A_{2}$ onto an annulus $A_{2}^{\prime}$ about $C_{2}^{\prime}$. Require $f_{2}$ to map $L_{1}$ onto a line segment $L_{1}^{\prime}$ on $\theta=0$ and to map $L_{2}$ onto a line segment $L_{2}^{\prime}$ on $\theta=\frac{\pi}{3}$. Note that $h f_{2}=f_{2} h$ on $A_{2}$.

Let $B_{2}^{\prime}=f_{2}\left(B_{2}\right)$. Use straight lines through the origin to divide $B_{2}^{\prime}$ into a number $n_{2}$ of congruent pieces. With these pieces as links, $B_{2}^{\prime}$ is a chain. The inverses under $f_{2}$ of these links are the links of $B_{2}$. The number $n_{2}$ should be chosen so that (as chains with open sets as links) $B_{2}$ is crooked in $A$, any three consecutive links of $B_{2}$ lie in a link of $A$, and each link of $B_{2}$ has small diameter.

By rotation of $\frac{\pi}{3}$, divide all of $A_{2}^{\prime}$, and hence $A_{2}$, into links. Each of $A_{2}$ and $A_{2}^{\prime}$ will then have $6 n_{2}$ links, and $A_{2}$ is crooked in $A$. Let $h_{2}^{\prime}$ be the rotation of 
the plane that takes each link of $A_{2}^{\prime}$ onto its successor; formally, $h_{2}^{\prime}$ is the rotation through the angle $2 \pi / 6 n_{2}$. Note that $\left(h_{2}^{\prime}\right)^{n_{2}}=h$.

Let $h_{2}=\left(f_{2}\right)^{-1} \circ h_{2}^{\prime} \circ f_{2}$ be defined on $A_{2}$. The homeomorphism $h_{2}$ is a "rotation" of $A_{2}$. It moves each link of $A_{2}$ to its successor link. The crux of the matter again is that $\left(h_{2}\right)^{n_{2}}=h$.

To construct the pseudocircle by induction, it is sufficient to describe a certain embedding of an annulus in $A_{2}$. Embed a simple closed curve $C_{3}$ into the annulus $A_{2}^{\prime}$ in the same manner that $C_{2}$ was embedded in $A$. The curve $C_{3}$ will be the union of $6 n_{2}$ arcs, each beginning in some link of $A_{2}$ and ending in its successor link. Each of these arcs will be the union of two crooked arcs, one of which winds once around the origin in a counterclockwise direction, as did $P_{1}$, and the other returning to the adjacent link, as did $P_{2}$. No two of these $6 n_{2}$ arcs will intersect, except possibly in an endpoint. The rotation $h_{2}^{\prime}$ will permute these arcs, so that $C_{3}$ is invariant under $h_{2}^{\prime}$.

The annular neighborhood $A_{3}$ of $C_{3}$ will be homeomorphic by $f_{3}$ to an annulus $A_{3}^{\prime}$ and will have $6 n_{2} n_{3}$ links, appropriately selected. The rotation $h_{3}^{\prime}$ is defined as rotation through the angle $2 \pi / 6 n_{2} n_{3}$. It has the property that $\left(h_{3}^{\prime}\right)^{n_{3}}=h_{2}^{\prime}$.

The inverse of $f_{2}$ takes $A_{3}$ onto an embedded annulus in $A$ (also called $A_{3}$ ). The homeomorphism $h_{3}=\left(f_{2} \circ f_{3}\right)^{-1} \circ h_{3}^{\prime} \circ\left(f_{2} \circ f_{3}\right)$ will be a "rotation" of $A_{3}$, and $\left(h_{3}\right)^{n_{3}}=h_{2}$.

The pseudocircle $X$ is defined as $\bigcap_{n=1}^{\infty} A_{n}$, where $A_{1}=A$ and $A_{n}$ is defined above.

THEOREM 1. There exists an uncountable abelian group $G$ of homeomorphisms of the pseudocircle $X$.

PROOF. Let $h_{1}=h, h_{2}, h_{3}, \ldots$ be the "rotations" (described above) constructed with the pseudocircle $X$. Each $h_{n}$ generates a finite cyclic group $R_{n}$ of $H(X)$; furthermore, there are inclusions $R_{1} \subset R_{2} \subset \cdots$. The direct limit of $\left\{R_{n}\right\}$ yields a countable abelian subgroup of $H(X)$ containing each $R_{n}$. Let $G$ be the closure of this group in $H(X)$; then $G$ is also an abelian group. Furthermore, since the identity map of $X=\lim _{n \rightarrow \infty} h_{n}$ and since $G$ is homogeneous, it follows that $G$ is perfect and hence uncountable.

A continuum $Z$ is said to be nearly homogeneous if given a point $z$ in $Z$ and a nomempty open set 0 of $Z$, there exists a homeomorphism of $Z$ onto itself taking $z$ into 0 .

THEOREM 2. The pseudocircle is nearly homogeneous.

PrOOF. The orbit of a point under $G$ is dense in $X$.

THEOREM 3. If $h: X \rightarrow X$ is a homeomorphism of the pseudocircle and $L$ is a composant of $X$ such that $h(L)=L$, then $h$ has a fixed point in $L$.

Proof. Let $x$ be some point of $L$. Since $x$ and $h(x)$ belong to $L$, there exists an irreducible continuum $Z$ containing $x$ and $h(x)$. The continuum $Z$ is either a point or a pseudoarc. Since the continuum $Z \cup h(Z)$ is indecomposable, either $h(Z) \subset Z$ or $Z \subset h(Z)$. Assume $h(Z) \subset Z$ (if not, replace $h$ with $h^{-1}$ ). Let $N=\bigcap_{n=1}^{\infty} h^{n}(Z)$. Hence $N$ is a subcontinuum of $Z$ satisfying $h(N)=N$. Since $N$ is either a point or a pseudoarc, there exists a point in $N$ fixed by $h$. 
THEOREM 4. The orbit of a point of $X$ under $G$ is a dense subset of $X$ intersecting uncountably many composants of $X$.

Proof. We show that uncountably many members of $G$ do not map a fixed composant of $X$ to the same composant.

Consider the uncountable collection $K=\left\{g \in G: g=\lim g_{i}\right.$, where $g_{i}=h_{1}^{K}$ 。 $h_{2}^{K_{2}} \circ \cdots \circ h_{i}^{K_{i}}$ and $K_{i}=0$ or 2$\}$. Since any three consecutive (open) links of $A_{n}$ are contained in an (open) link of $A_{n-1}$, each lim $g_{i}$ does exist. Furthermore, the only homeomorphism of $K$ that fixes a point is the identity. In fact, no two homeomorphisms of $K$ map a fixed composant of $X$ to the same composant.

3. Almost chainable continua without a dense $G_{\delta}$-orbit. A continuum is almost chainable if, for each positive $\varepsilon$, there exists an $\varepsilon$-covering $D$ of $X$ and a chain $C=\left\{C_{1}, \ldots, C_{n}\right\}$ of elements of $D$ such that no $C_{i}(1<i \leq n)$ intersects an element of $D-C$ and every point of $X$ is within $\varepsilon$ of some point of $C^{*}$. The set $C_{n}$ is called an end link of $C$.

C. E. Burgess introduced this fruitful notion in [2] and proved that the pseudocircle is almost chainable.

In this section we give sufficient conditions for an almost chainable continuum to fail to have a dense $G_{\delta}$-orbit. The pseudocircle satisfies these conditions by $[\mathbf{2}$, 14], and the construction of $\S 1$, and so it does not have a dense $G_{\delta}$-orbit.

Our theorem generalizes a theorem of Hagopian [7], who used almost chainability to provide another proof that the pseudocircle is not homogeneous.

A continuum $X$ is said to have property $K$ at a point $a$ of $X$ provided that, given $\varepsilon>0$, there exists a $\delta>0$ such that if $b$ is in $X$ with $d(a, b)<\delta$ and $A$ is a subcontinuum of $X$ containing $a$, then there is a subcontinuum $B$ of $X$ containing $b$ such that the Hausdorff distance from $A$ to $B$ is less than $\varepsilon$. A continuum has property $K$ if it has property $K$ at each point.

THEOREM 5. Suppose $X$ is an almost chainable continuum that has property $K$. If $X$ has a homeomorphism without any fixed point, then $X$ does not have a dense $G_{\delta}$-orbit.

ProOF. Let $f: X \rightarrow X$ be a homeomorphism without a fixed point. Let $\varepsilon$ be a positive number such that $d(f(x), x)>2 \varepsilon$ and $d\left(f^{-1}(x), x\right)>2 \varepsilon$.

Suppose the theorem is not true, so that $X$ has a dense $G_{\delta}$-orbit $A$. We can apply the Effros theorem [4] to obtain a countable collection $W$ of open sets of $X$ such that (1) $A \subset W^{*}$; and (2) $W$ has the $\varepsilon$-push property with respect to $A$, that is, if $W \in \mathcal{W}$ and $x, y \in A \cap W$, then there is some homeomorphism $h$ in $H(X)$ such that $h(x)=y$ and $h$ moves no point of $X$ more than $\varepsilon$.

For each $j$ in $N$, there exists a $\frac{1}{j}$-cover $D_{j}$ of $X$ and a chain $C_{j}=\{C(j, i) \mid 1 \leq i \leq$ $\left.n_{j}\right\}$ of elements of $D_{j}$ such that no element of $D_{j}-C_{j}$ intersects $\left\{C(j, i) \mid 2 \leq i \leq n_{j}\right\}^{*}$ and every point of $X$ is within $\frac{1}{j}$ of some element of $C_{j}^{*}$.

For each $j$ in $N$, choose a point $p_{j}$ in $C\left(j, n_{j}\right)$, and let $K_{j}$ denote the $p_{j}$ component of $X-C(j, 1)$. Without loss of generality assume that $p_{1}, p_{2}, \ldots$ converges to the point $p$ of $X$, and note that the sequence $K_{1}, K_{2}, \ldots$ of continua converges to $X$.

Choose a point $x$ in $A-\{p\}$, and then choose a sequence $x_{1}, x_{2}, \ldots$ of points of $K_{i}$ that converges to $x$. For each $i$ there is some $r_{i}$ such that $x_{i} \in C\left(i, r_{i}\right)$. Use 
the fact that $X$ has property $K$ at $x_{i}$ to find a continuum $\hat{K}_{i}$ such that (1) $\hat{K}_{i}$ is within $\frac{1}{i}$ of $K_{i}$ with respect to the Hausdorff metric, (2) there is a point $\hat{x}_{i}$ in $A \cap \hat{K}_{i} \cap C\left(i, r_{i}\right),(3) \hat{K}_{i} \subset C_{i}^{*}$, and (4) $\hat{K}_{i} \cap C(i, j) \neq \varnothing$, for $1<j \leq n_{i}$.

Choose $\hat{p}_{i} \in \hat{K}_{i} \cap C\left(i, n_{i}\right)$. Note that $\hat{x}_{i}, \hat{x}_{2}, \ldots$ converges to $x, \hat{p}_{1}, \hat{p}_{2}, \ldots$ converges to $p$, and $\hat{K}_{1}, \hat{K}_{2}, \ldots$ converges to $X$. There is some open set $U$ such that $x \in U$, $U \cap f(U)=\varnothing$, and both $U$ and $f(U)$ are subsets of some sets $W$ and $W^{\prime}$ in $\mathcal{W}$. There is some $j$ in $N$ such that (1) $x_{j}$ and $\hat{x}_{j}$ are in $U,(2) C\left(j, r_{j}\right) \subset U,(3) \frac{1}{j}<\varepsilon$, and (4) there is an $m_{j}$ such that $C\left(j, m_{j}\right) \subset f(U)$. For simplicity, let $r_{j}=n$, $m_{j}=m$.

Either $n<m$ or $m<n$. Suppose first that $n<m$. Since $f\left(\hat{x}_{j}\right) \in f(U)$, there is a homeomorphism $h$ in $H(X)$ such that $h f\left(\hat{x}_{j}\right) \in C(j, m)$ and $h$ moves no point of $X$ more than $\varepsilon$. Note that $h f$ moves each point of $X$ a distance greater than $\varepsilon$.

For $1 \leq k \leq n_{j}$, let

$$
A_{k}=\left\{y \in \hat{K}_{j} \cap C(j, k) \mid h f(y) \in\{C(j, i) \mid k \leq i \leq n\}^{*}\right\}
$$

and

$$
B_{k}=\left\{y \in \hat{K}_{j} \cap C(j, k) \mid h f(y) \notin\{C(j, i) \mid k \leq i \leq n\}^{*}\right\} .
$$

Let $A=\bigcup\left\{A_{k}: k=1, \ldots, n_{j}\right\}$ and $B=\bigcup\left\{B_{k}: k=1, \ldots, n_{j}\right\}$. Then $\hat{x}_{j} \in A$ and $\hat{p}_{j} \in B, A \cup B=\hat{K}_{j}$, and $A$ and $B$ are mutually exclusive closed point sets. Since this is a contradiction, it must be the case that $m<n$.

Since $C(j, m) \cap \hat{K}_{j} \neq \varnothing$, we can use property $K$ again to obtain a continuum $L_{j}$ of $X$ such that (1) $L_{j} \subset C_{j}^{*}$, (2) $L_{j} \cap C(j, m) \cap A \neq \varnothing$, and (3) $L_{j}$ intersects each link of $C_{j}-\{C(j, 1)\}$. Choose $q_{j} \in L_{j} \cap C\left(j, n_{j}\right)$. Suppose $z \in L_{j} \cap C(j, m) \cap A$. There is $h^{\prime}$ in $H(X)$ such that $h^{\prime} f^{-1}(z) \in C(j, n)$ and $h^{\prime}$ moves no point of $X$ more than $\varepsilon$.

For $1 \leq k \leq n_{j}$, let

$$
A_{k}^{\prime}=\left\{y \in L_{j} \cap C(j, k) \mid h^{\prime} f^{-1}(y) \in\{C(j, i) \mid k \leq i \leq n\}^{*}\right\}
$$

and let

$$
B_{k}^{\prime}=\left\{y \in L_{j} \cap C(j, k) \mid h^{\prime} f^{-1}(y) \notin\{C(j, i) \mid k \leq i \leq n\}^{*}\right\} .
$$

Let $A^{\prime}=\bigcup A_{k}^{\prime}$ and $B=\bigcup B_{k}^{\prime}$. Then $z \in A^{\prime}, q_{j} \in L_{j} \cap C\left(j, n_{j}\right) \subset B^{\prime}$, and $A^{\prime}$ and $B^{\prime}$ are disjoint closed point sets whose union is $L_{j}$. Again we have a contradiction, and it follows that $X$ has no dense $G_{\delta}$-orbit.

4. Homeomorphic open sets of the pseudoarc and the pseudocircle. A cover $\mathcal{U}$ of a compactum is said to be taut if $\bar{U} \cap \bar{U}^{\prime} \neq \varnothing, U$ and $U^{\prime} \in \mathcal{U}$, implies $U \cap U^{\prime} \neq \varnothing$.

The notion of a pattern, defined next, is used to describe the way one chain is embedded in another. If $A$ and $B$ are subsets of the nonnegative integers, then a function $f: A \rightarrow B$ is a pattern if whenever $i, i+1 \in A$, then $|f(i+1)-f(i)| \leq 1$. If $\mathcal{V}=\left\{V_{i} \mid i \in A\right\}$ and $\mathcal{U}=\left\{U_{i} \mid i \in B\right\}$ are collections of open sets of the compactum $X$, then $\mathcal{V}$ follows the pattern $f$ in $U$ provided $V_{i} \subseteq U_{f(i)}$ for each $i \in A$. We will call $f$ a pattern on $\mathcal{U}$.

We also must describe the way one finite collection of chains is embedded in another such collection. To do this, we must speak of a pattern that a collection of chains follows in another collection of chains. In such a pattern, there are two 
subscripts; the first indicates a chain and the second indicates a link of that chain. Here is the definition of such a pattern: If $A$ and $B$ are two subsets of $(N \cup\{0\}) \times$ $(N \cup\{0\})$, then the function $f: A \rightarrow B$ is said to be a pattern provided that (1) whenever $(i, k),\left(i, k^{\prime}\right) \in A, f(i, k)_{1}=f\left(i, k^{\prime}\right)_{1}$ (the first coordinates are the same); and (2) whenever $(i, k),(i, k+1) \in A,\left|f(i, k+1)_{2}-f(i, k)_{2}\right| \leq 1$ (the second coordinates differ by no more than 1$)$.

If $\mathcal{V}=\left\{V_{(i, j)} \mid(i, j) \in A\right\}$ and $\mathcal{U}=\left\{U_{(i, j)} \mid(i, j) \in B\right\}$ are collections of open sets of the compactum $X$, then $\mathcal{V}$ follows the pattern $f$ in $\mathcal{U}$ provided $V_{(i, j)} \subseteq U_{f(i, j)}$ for each $(i, j) \in A$. Again $f$ is a pattern on $U$. We write $V_{i j}$ for $V_{(i, j)}$.

THEOREM 6. Let $U_{1}, U_{2}, \ldots$ and $V_{1}, V_{2}, \ldots$ be sequences of finite collections of chains in the compact metric spaces $X$ and $Y$, respectively, such that for each $i$

(1) $U_{i}$ and $V_{i}$ consist of a finite collection of taut chains such that if $C$ and $C^{\prime}$ are two different chains in $U_{i}$, then $C^{*} \cap C^{\prime *}=\varnothing$ in $X$, and if $D$ and $D^{\prime}$ are two different chains in $V_{i}$, then $D^{*} \cap D^{* *}=\varnothing$;

(2) $U_{i}^{* *}=X, V_{i}^{* *}=Y$;

(3) $\lim _{i} \operatorname{mesh} U_{i}=\lim _{i} \operatorname{mesh} V_{i}=0$;

(4) the number of chains in $U_{i}$ is the same as the number of chains in $V_{i}$ and if $U(i, 1), \ldots, U\left(i, n_{i}\right)$ is a listing of the chains in $U_{i}$, we can list the chains in $V_{i}=V(i, 1), \ldots, V\left(i, n_{i}\right)$ so that for each $j,|U(i, j)|=|V(i, j)|$;

(5) both $U_{i+1}$ and $V_{i+1}$ follow a pattern $f_{i}$ in $U_{i}$ and $V_{i}$, respectively, with respect to the listings in (4);

(6) each link of each chain of $U_{i}$ contains a point of $X$ not contained in any other link of a chain $U_{i}$ (and similarly for $V_{i}$ and $Y$ ).

Then there exists a homeomorphism $h$ taking $X$ onto $Y$.

Proof. For each $j, 1 \leq j \leq n_{i}$, denote the chain $U(i, j)$ by $\{U(i, j, 0), \ldots$, $\left.U\left(i, j, a_{i, j}\right)\right\}$ and the chain $V(i, j)$ by $\left\{V(i, j, 0), \ldots, V\left(i, j, a_{i, j}\right)\right\}$. For each $i$, define $h_{i}: U_{i}^{* *} \rightarrow V_{i}^{* *}$ by $h_{i}: U(i, j, k)=V(i, j, k)$ for $1 \leq j \leq n_{i}, 0 \leq k \leq a_{i j}$. Note that $h_{i}$ is one-to-one and onto, and that $h_{i}$ takes adjacent links of chains of $U_{i}$ to adjacent links of chains of $V_{i}$.

From $f_{i}$ define $\hat{f}_{i}: U_{i+1} \rightarrow U_{i}$ and $f_{i}^{\prime}: V_{i+1} \rightarrow V_{i}$ by $\hat{f}_{i}(U(i+1, j, k))=U(i, l, m)$ if $f_{i}(j, k)=(l, m)$ and $f_{i}^{\prime}(V(i+1, j, k))=V(i, l, m)$ if $f_{i}(j, k)=(l, m)$.

For each $x$ in $X$, there is a sequence $C(1, x), C(2, x), \ldots$ such that (1) for each $i, x \in C(i, x)$ and $C(i, x) \in U_{i}^{*}$, and $(2) \hat{f}_{i}(C(i+1, x))=C(i, x)$. Then $\{x\}=$ $\bigcap_{i} C(i, x)$ and $\bigcap_{i} \overline{h_{i} C(i, x)}$ is nonempty and degenerate. Note that $f_{i-1}^{\prime} h_{i}(C(i, x))$ $=h_{i-1} \hat{f}_{i-1}(C(i, x))$. Then define $h(x)=\bigcap_{i} \overline{h_{i}(C(i, x))}$. We shall prove $h$ is a homeomorphism.

The function $h$ is injective. Let $x$ and $x^{\prime}$ be points of $X$. There exists an integer $i$ such that if $L$ is a link of a chain of $U_{i}$ containing $x$, and $L^{\prime}$ is a link of a chain of $U_{i}$ containing $x^{\prime}$, then the closures of $L$ and $L^{\prime}$ are disjoint. Hence the closures of $h_{i}(L)$ and $h_{i}\left(L^{\prime}\right)$ are disjoint. Therefore $h(x) \neq h\left(x^{\prime}\right)$.

The function $h$ is continuous. If $y$ is a point of $h(X)$, then $y=\bigcap_{i} \overline{h_{i} C(i, x)}$ for some $x$ in $X$. Let $O$ be an open set in $Y$ containing $y$. We will show that there exists an integer $i$ such that $h_{i}(C(i, x)) \subset O$.

Choose $i$ so large that the closure of $h(C(i, x))$ as well as the closure of any link adjacent to $h_{i}(C(i, x))$ is contained in $O$. If $z \in C(i, x)$, then $C(i, z)=C(i, x)$ or a link adjacent to $C(i, x)$. In either case $\overline{h_{i}(C(i, z))} \subset O$. Hence $h(z) \in O$. 
The function $h$ is surjective. It suffices to show $h(X)$ is dense in $Y$. Suppose there exists a link $K$ of a chain of $V_{i}$ such that $\mathrm{Cl}(K) \cap h(X)=\varnothing$. Let $L$ be the link of the chain $U_{i}$ such that $h_{i}(L)=K$. Condition (6) of the hypothesis implies that $L$ contains a point $x$ such that $C(i, x)=L$. Hence $h_{i}(C(i, x))=K$. Therefore $h(x) \in \mathrm{Cl}(K)$. This is a contradiction.

THEOREM 7. Suppose $X$ is the pseudoarc and $Y$ is the pseudocircle. Then there are open sets $O$ of $X$ and $U$ of $Y$ and a homeomorphism $h: \bar{O} \rightarrow \bar{U}$ such that $h(\partial O)=\partial U$.

PROOF. Suppose that $O=O_{1}, O_{2}, \ldots$ is a defining sequence of taut chain covers of $X$ such that for each $i(1)$ mesh $O_{i}<2^{-i}$, and (2) $O_{i}$ is an amalgamation of $O_{i+1}$. Suppose that $U=U_{1}, U_{2}, \ldots$ is a defining sequence of taut circular chain covers of $Y$ such that for each $i$, (1) mesh $U_{i}<1 / 2^{i}$, and $(2) U_{i}$ is an amalgamation of $U_{i+1}$. Without loss of generality, assume that $O_{1}$ and $U_{1}$ both have at least three links.

Now choose $O \in O_{1}$ such that $O$ is not an end link, and choose $U \in \mathcal{U}_{1}$. For each $i$, let $C_{i}=\left\{O^{\prime} \in O_{i+1} \mid O^{\prime} \subseteq O\right\}$ and let $D_{i}=\left\{U^{\prime} \in \mathcal{U}_{i+1} \mid U^{\prime} \subseteq U\right\}$. Note that $C_{i}^{*}=O$ and $D_{i}^{*}=U$.

It is the case that if $x \in \bar{O}-O$ and $i \in N$, then $x$ is in the closure of exactly one link $C$ of $C_{i}$ because if $x \in \bar{C} \cap \bar{C}^{\prime}$, where both $C$ and $C^{\prime}$ are in $C_{i}$, then $x \in C \cup C^{\prime}$ (tautness) and so $x \in O$.

Thus for each $i, C_{i}^{\prime}=\left\{C \cup(\bar{C} \cap(\bar{O}-O)) \mid C \in C_{i}\right\}$ is an open cover of $\bar{O}$ consisting of a finite collection of maximal chains. For each $i$, let $C(i)$ denote the set of all maximal chains in $C_{i}^{\prime}$ and let $D(i)$ denote the set of all maximal chains in $D_{i}^{\prime}=\left\{D \cup(\bar{D} \cap(\bar{U}-U)) \mid D \in D_{i}\right\}$. Then list the chains in each of $C(i)$ and $D(i): C(i)=\left\{C(i, 1), \ldots, C\left(i, n_{i}\right)\right\}$ and $D(i)=\left\{D(i, 1), \ldots, D\left(i, m_{i}\right)\right\}$; and list the links in each chain (in order): for $1 \leq j \leq n_{i}, C(i, j)=\left\{C(i, j, 0), \ldots, C\left(i, j, a_{i, j}\right)\right\}$, and for $1 \leq j \leq m_{i}, D(i, j)=\left\{D(i, j, 0), \ldots, D\left(i, j, b_{i, j}\right)\right\}$. Note that

(1) $C(i, j)^{*} \cap C\left(i, j^{\prime}\right)^{*}=\varnothing$ if $j \neq j^{\prime}$

(2) $C(i, j)^{*}$ is open and closed in $\bar{O}$;

(3) $C(i, j)^{*}$ contains a continuum $Z_{i j}$ such that $Z_{i j} \cap(C(i, j, 0)-O) \neq \varnothing$,

$$
Z_{i j} \cap\left(C\left(i, j, a_{i, j}\right)-O\right) \neq \varnothing, \quad \text { and } Z_{i j} \subseteq \bar{O} .
$$

Analogous properties hold for the $D(i, j)$ 's.

The idea of the proof is the following: We will construct a sequence $\hat{C}(1), \hat{C}(2), \ldots$ of open covers of $\bar{O}$ such that each $\hat{C}(i)$ consists of a finite collection of taut maximal chains, and a sequence $\hat{D}(1), \hat{D}(2), \ldots$ of open covers of $\bar{U}$ such that each $\hat{D}(i)$ consists of a finite collection of taut maximal chains. Further, for each $i$ there will be a pattern $f_{i}$ such that $\hat{C}(i+1)$ follows $f_{i}$ in $\hat{C}(i)$, and $\hat{D}(i+1)$ follows $f_{i}$ in $\hat{D}(i)$. We will also make sure that the hypotheses of the previous theorem are satisfied by the sequences $\hat{C}(1), \hat{C}(2), \ldots$ and $\hat{D}(1), \hat{D}(2), \ldots$ and then we will apply this theorem to conclude that $\bar{O}$ is homeomorphic to $\bar{U}$.

Now define $\hat{C}(1)$ to be $C(1)$ : so for $1 \leq j \leq n_{1}$ and $0 \leq k \leq a_{1, j}, \hat{C}(1, j, k)=$ $C(1, j, k)$ and $\hat{C}(1, j)=C(1, j)$. Let $N_{1}=n_{1}$ and $A_{1, j}=a_{1, j}$.

There is an integer $k_{1}$ such that $D\left(k_{1}\right)$ consists of at least $N_{1}$ chains and, for some $N_{1}$ chains in $D\left(k_{1}\right)$, each of these chains has at least $M$ links, where $M=$ $\max \left\{|\hat{C}(i, j)|: j \leq N_{1}\right\}$. Assume without loss of generality that these $N_{1}$ chains are listed first in $D\left(k_{1}\right)$. 
In the next three paragraphs, we define $\hat{D}(1)$. The first link of the first chain of $\hat{D}(1)$ is the first link of the first chain of $D\left(k_{1}\right)$. Formally $\hat{D}(1,1,1)=D\left(k_{1}, 1,1\right)$. Similarly, the second link $\hat{D}(1,1,2)$ is defined to be $D\left(k_{1}, 1,2\right)$. A similar definition is used until we get to the last link $\hat{D}\left(1,1, A_{1,1}\right)$ of the first chain of $\hat{D}(1)$, which is defined to be the union of $D\left(k_{1}, 1, A_{1,1}\right)$ and any other links of the first chain of $\hat{D}(1)$ that follow it.

The links of the second chain of $\hat{D}(1)$ are defined similarly. In fact, a similar definition is used until we get to the last link of the last chain of $\hat{D}(1)$ : this link is $\hat{D}\left(1, N_{1}, A_{1, N_{1}}\right)$ and is defined as the union of $D\left(k_{1}, N_{1}, A_{1, N_{1}}\right)$ and all the links of $D\left(k_{1}, N_{1}\right)$ that follow it and all the links of all the chains of $D\left(k_{1}\right)$ that have not yet been considered.

Define $\hat{D}(1)=\left\{\hat{D}(1,1), \hat{D}(1,2), \ldots, \hat{D}\left(1, N_{1}\right)\right\}$, where $\hat{D}(1, i)$ is the $i$ th chain of $\hat{D}(1)$.

There is some $k_{2}>k_{1}$ such that $D\left(k_{2}\right)$ closure-refines $\hat{D}(1)$, mesh $D\left(k_{2}\right) \leq \frac{1}{4}$, and $2^{-k_{2}-1}$ is less than the distance between $\partial U$ and

$$
\overline{\left\{\hat{D}(1, i, j) \mid 1 \leq i \leq N_{1}, 0<j<A_{1,1}\right\}^{*}} .
$$

Define $\hat{D}(2)=D\left(k_{2}\right)$. Hence, for $1 \leq j \leq m_{k_{2}} \equiv M_{2}$ and $0 \leq l \leq b_{k_{2} j} \equiv B_{2 j}$, we have $\hat{D}(2, j, l)=D\left(k_{2}, j, l\right)$.

Now $\hat{D}(2)$ follows some pattern $f_{1}$ in $\hat{D}(1)$ so that $f_{1}: \alpha \rightarrow \beta$, where

$$
\begin{aligned}
& \alpha=\left\{(i, j) \mid 1 \leq i \leq M_{2}, 0 \leq j \leq B_{2 i}\right\}, \\
& \beta=\left\{\left(i^{\prime}, j^{\prime}\right) \mid 1 \leq i^{\prime} \leq N_{1}, 0 \leq j^{\prime} \leq A_{1 i^{\prime}}\right\} .
\end{aligned}
$$
let

We must construct $\hat{C}(2)$ so that it follows $f_{1}$ in $\hat{C}(1)$. To this end, for $1 \leq i \leq M_{2}$,

$$
F_{i}=\left\{\hat{D}(i, j, l) \mid \text { there is } k \text { such that }(i, k) \text { is in } \alpha \text { and } f_{1}(i, k)=(j, l)\right\} .
$$

Thus $F_{i}$ is a subchain of $\hat{D}(1, j)$ that contains either the first link or the last link of $\hat{D}(1, j)$.

There is a sequence $G_{1}, G_{2}, \ldots, G_{M_{2}}$ of mutually exclusive open and closed point sets of $\bar{O}$ such that

(4) $\bar{O}=G_{1} \cup G_{2} \cup \cdots \cup G_{M_{2}}$;

(5) there is $l$ in $N$ such that each $G_{i}$ is a union of some of the links in $C(l)^{*}$;

(6) if, for each $i, H_{i}=\left\{\hat{C}(1, j, k) \in \hat{C}(1, j) \mid \hat{D}(1, j, k) \in F_{i}\right\}$ (where $G_{i} \subset$ $\left.\hat{C}(1, j)^{*}\right)$, then $G_{i} \subseteq H_{i}^{*}$; and

(7) $H_{i}$ essentially covers $G_{i}$.

To see that such a sequence of open sets exists, consider the following: There is a pseudoarc $P$, a proper subcontinuum of $X$, such that

(8) some subcontinuum $P^{\prime}$ of $P$ is a subset of $\bar{O}$,

(9) $P \cap \bar{O} \subseteq \hat{C}(1,1)^{*}$,

(10) $\hat{C}(1,1,0) \cap P^{\prime}$ contains a limit point of $P-\bar{O}$, and

(11) $\hat{C}\left(1,1, A_{1,1}\right) \cap P^{\prime}$ contains a limit point of $P-\bar{O}$.

Without loss of generality, suppose that $\hat{D}(2,1), \ldots, \hat{D}(2, n)$ is the list of all the chains $D$ in $\hat{D}(2)$ such that $D^{*} \subseteq \hat{D}(1,1)^{*}$, and that associated with $\hat{D}(2,1)$ is a continuum $Z$ that "runs all the way through $\hat{D}(1,1)^{*}$," i.e., there is some 
subcontinuum $Z^{\prime}$ of $Z$ such that $Z^{\prime} \subseteq \hat{D}(2,1)^{*}$ and $Z^{\prime} \cap(\bar{U}-U) \cap \hat{D}(1,1,0) \neq \varnothing$ and $Z^{\prime} \cap(\bar{U}-U) \cap \hat{D}\left(1,1, A_{1,1}\right) \neq \varnothing$.

Choose $n$ mutually exclusive pseudoarcs $P_{1}, \ldots, P_{n}$ contained in $P$ such that for each $i$

(12) some subcontinuum $P_{i}^{\prime}$ of $P_{i}$ is a subset of $\hat{C}(1,1)^{*}$,

(13) $P_{i} \not \subset \hat{C}(1,1)^{*}$ (this will be redundant),

(14) $\hat{C}(1,1,0) \cap P_{i}^{\prime}$ contains a limit point of $P-\bar{O}$, and

(15) $\hat{C}\left(1,1, A_{1,1}\right) \cap P_{i}^{\prime}$ contains a limit point of $P-\bar{O}$.

There is an integer $k$ such that $2^{-k}<\frac{1}{2} \min \left\{d\left(P_{i}, P_{j}\right) \mid i \neq j\right\}$. For each $i$ there is an integer $k_{i}^{\prime}>k$ such that

(16) some chain $E_{i}$ of $O_{k_{i}^{\prime}}$ essentially covers some subcontinuum of $P_{i}$,

(17) neither end link of $E_{i}$ intersects $\bar{O}$,

(18) $\hat{C}(1,1,0) \cap \partial O$ contains a limit point of $E_{i}^{*}-\bar{O}$, and

(19) $\hat{C}\left(1,1, A_{1,1}\right) \cap \partial O$ contains a limit point of $E_{i}^{*}-\bar{O}$.

Note that $E_{i}^{*} \cap E_{j}^{*}=\varnothing$ if $i \neq j$.

Use the crookedness of the chains covering $X$ to find a $k_{i}>k_{i}^{\prime}$ such that there is a subchain $L_{i}$ of $O_{k_{i}}$ such that

(20) neither end link of $L_{i}$ intersects $\bar{O}$,

(21) $L_{i}^{*} \cap \bar{O} \subseteq H_{i}^{*}$,

(22) each link of $H_{i}$ contains a link of $L_{i}$ which does not intersect any other link of $H_{i}$,

(23) $L_{i}^{*} \subset E_{i}^{*}$

(24) $\hat{D}(2, i)^{*} \cap \hat{D}(1,1,0) \cap \partial U \neq \varnothing$ if and only if some point of $\hat{C}(1,1,0) \cap \partial O$ is a limit point of $L_{i}^{*}-\bar{O}$, and

(25) $\hat{D}(2, i)^{*} \cap \hat{D}\left(1,1, A_{1,1}\right) \cap \partial U \neq \varnothing$ if and only if some point of $\hat{C}\left(1,1, A_{1,1}\right) \cap \partial O$ is a limit point of $L_{i}^{*}-\bar{O}$.

For $i>1$, define $G_{i}=L_{i}^{*} \cap \bar{O}$ and let $G_{1}=\hat{C}(1,1)^{*}-\left\{G_{i} \mid i>1\right\}^{*}$.

We have thus obtained $G_{1}, G_{2}, \ldots, G_{n}$, and still need to get the $G_{i}$ 's contained in the other chains of $\hat{C}(1)$. But we can do this in exactly the same way. Choose $l>k_{i}$, for all $i$.

Now for each $i, 1 \leq i \leq M_{2}$, denote $H_{i}$ by $\left\{\hat{C}\left(1, j, \alpha_{i}\right), \ldots, \hat{C}\left(1, j, \beta_{i}\right)\right\}$ (where $\left.H_{i}^{*} \subseteq \hat{C}(1, j)^{*}\right)$.

For $1 \leq i \leq M_{2}, \alpha_{i} \leq k \leq \beta_{i}$, let $g(i, k)=G_{i} \cap \hat{C}(1, j, k)$ and let $g_{i}=$ $\left\{g\left(i, \alpha_{i}\right), \ldots, g\left(i, \beta_{i}\right)\right\}$. The chain $g_{i}$ is an open taut cover of the hereditarily indecomposable compactum $G_{i}$. Also each link in $g_{i}$ is a union of links of $C(l)^{*}$.

For each $i$, let $f_{i 1}=f_{1} \mid\left\{(i, j) \mid 0 \leq j \leq B_{2, i}\right\}$. Next we need to find an open taut cover $V_{i}$ of $G_{i}$ such that only end links of $V_{i}$ contain boundary points of $\bar{O}$ and such that $V_{i}$ follows $f_{i 1}$ in $g_{i}$ (because $\hat{D}(2, i)$ follows $f_{i 1}$ in $\hat{D}(1, j)$, where $\left.\hat{D}(2, i)^{*} \subset \hat{D}(1, j)^{*}\right)$. We indicate how to do this in the most involved case and leave the other cases to the reader.

For convenience, assume $\hat{D}(2, i)^{*} \subset \hat{D}(1,1)^{*}$.

Suppose

(26) $g\left(i, \alpha_{i}\right) \cap \partial O \neq \varnothing, g\left(i, \beta_{i}\right) \cap \partial O \neq \varnothing$,

(27) $\alpha_{i} \neq \beta_{i}$

(28) $\left(f_{i 1}\right)^{-1}(1,0)$ is not degenerate, and 
(29) $\left(f_{i 1}\right)^{-1}\left(1, A_{1,1}\right)$ is not degenerate.

There are open sets $g^{\prime}\left(i, \alpha_{i}-1\right)$ and $g^{\prime}\left(i, \alpha_{i}\right)$ such that

(30) $g\left(i, \alpha_{i}\right) \cap \partial O \subseteq g^{\prime}\left(i, \alpha_{i}-1\right)$,

(31) $\overline{g^{\prime}\left(i, \alpha_{i}\right)} \cap \partial O=\varnothing$,

(32) $g^{\prime}\left(i, \alpha_{i}-1\right) \cup g^{\prime}\left(i, \alpha_{i}\right)=g\left(i, \alpha_{i}\right)$, and

(33) $\overline{g^{\prime}\left(i, \alpha_{i}-1\right)} \cap \overline{g(i, k)}=\varnothing$, for all $k>\alpha_{i}$.

There are open sets $g^{\prime}\left(i, \beta_{i}+1\right)$ and $g^{\prime}\left(i, \beta_{i}\right)$ such that

(34) $g\left(i, \beta_{i}\right) \cap \partial O \subseteq g^{\prime}\left(i, \beta_{i}+1\right)$,

(35) $\overline{g^{\prime}\left(i, \beta_{i}\right)} \cap \partial O=\varnothing$,

(36) $g^{\prime}\left(i, \beta_{i}+1\right) \cup g^{\prime}\left(i, \beta_{i}\right)=g\left(i, \beta_{i}\right)$, and

(37) $\overline{g^{\prime}\left(i, \beta_{i}+1\right)} \cap \overline{g(i, k)}=\varnothing$, for all $k<\beta_{i}$.

Then $g_{i}^{\prime}=\left\{g^{\prime}\left(i, \alpha_{i}-1\right), g^{\prime}\left(i, \alpha_{i}\right), g\left(i, \alpha_{i}+1\right), \ldots, g\left(i, \beta_{i}-1\right), g^{\prime}\left(i, \beta_{i}\right), g^{\prime}\left(i, \beta_{i}+1\right)\right\}$ is a taut chain cover of $G_{i}$. Furthermore, only $g^{\prime}\left(i, \alpha_{i}-1\right)$ and $g^{\prime}\left(i, \beta_{i}+1\right)$ contain boundary points of $\bar{O}$. Let $g^{\prime}(i, j)=g(i, j)$, for $\alpha_{i}<j<\beta_{i}$.

Now go to $\hat{D}(1,1)$ and do the same thing. There are open sets $\hat{D}^{\prime}(1,1,-1)$ and $\hat{D}(1,1,0)$ such that

(38) $\hat{D}(1,1,0) \cap \partial U \subseteq \hat{D}^{\prime}(1,1,-1)$,

(39) $\overline{\hat{D}^{\prime}(1,1,0)} \cap \partial U=\varnothing$,

(40) $\hat{D}^{\prime}(1,1,-1) \cup \hat{D}^{\prime}(1,1,0)=\hat{D}(1,1,0)$,

(41) $\overline{\hat{D}^{\prime}(1,1,-1)} \cap \overline{\hat{D}(1,1, k)}=\varnothing$, for $k>0$, and

(42) $\hat{D}^{\prime}(1,1,-1)$ contains one end link of $\hat{D}(2, i)$ and no other link of $\hat{D}(2, i)$.

There are open sets $\hat{D}^{\prime}\left(1,1, A_{1,1}\right)$ and $\hat{D}^{\prime}\left(1,1, A_{1,1}+1\right)$ with similar properties with respect to $\hat{D}\left(1,1, A_{1,1}\right)$. Let

$$
\begin{aligned}
\hat{D}^{\prime}(1,1)=\left\{\hat{D}^{\prime}(1,1,-1), \hat{D}^{\prime}(1,1,0), \hat{D}(1,1,1), \ldots,\right. \\
\left.\hat{D}\left(1,1, A_{1,1}-1\right), \hat{D}^{\prime}\left(1,1, A_{1,1}\right), \hat{D}^{\prime}\left(1,1, A_{1,1}+1\right)\right\} .
\end{aligned}
$$

Let $\hat{D}^{\prime}(1,1, j)=\hat{D}(1,1, j)$, for $0<j<A_{1,1}$.

The chain $\hat{D}(2, i)$ follows a pattern $\hat{f}_{i 1}$ in $\hat{D}^{\prime}(1,1)$, where $\hat{f}_{i 1}(i, 0)$ is one of $(1,-1)$ or $\left(1, A_{1,1}+1\right), \hat{f}_{i 1}\left(i, B_{2 i}\right)$ is the other, and $\hat{f}_{i 1}(i, j)=f_{i 1}(i, j)$, for $0<j<B_{2 i}$. Let us say that $\hat{f}_{i 1}(i, 0)=(1,-1)$ and $\hat{f}_{i 1}\left(i, B_{2 i}\right)=\left(1, A_{1,1}+1\right)$ for convenience.

There exists a taut open chain cover $V_{i}=\left\{V(i, 0), \ldots, V\left(i, B_{2 i}\right)\right\}$ of $G_{i}$ such that $V_{i}$ follows the pattern $\hat{f}_{i 1}$ in $g_{i}^{\prime}$ (Theorem 3 of $[\mathbf{1 2}]$ ). Note that this means end links of $V_{i}$ are contained in end links of $g_{i}^{\prime}$; in fact, no other link of $V_{i}$ can contain a boundary point of $\bar{O}$. Moreover, $V_{i}$ follows the pattern $f_{i 1}$ in $g_{i}$. Finally, each end link of $V_{i}$ contains a boundary point of $O$ (additional manipulation of the same sort may be required to achieve this in the additional case).

We are almost ready to define $\hat{C}(2)$. Our only remaining problem is that the links of $V_{i}$ may not be unions of links of a defining chain.

Let $\delta$ be a positive number such that $2 \delta$ is less than the distance between the closures of any two nonadjacent links of $V_{i}$ and also $2 \delta$ is less than the distance between $\partial 0$ and the closure of any non-end link of $V_{i}$. There is some $l_{i}>l$ such that $2^{-l_{i}}<\delta$. For each $j, 0 \leq j \leq B_{2 i}$, let $V^{\prime}(i, j)=\left\{c \in C_{l_{i}}^{\prime} \mid c \subset g\left(i, f_{i 1}(i, j)_{2}\right)\right.$ and $c \cap V(i, j) \neq \varnothing\}^{*}$, where $f_{i 1}(i, j)_{2}$ denotes the second coordinate of $f_{i 1}(i, j)$. Since $g\left(i, f_{i 1}(i, j)_{2}\right)$ is a union of links of $C_{l}^{\prime}$ and, thus, of $C_{l_{i}}^{\prime}$, it follows that $V(i, j) \subseteq$ $V^{\prime}(i, j) \subseteq g\left(i, f_{i 1}(k, i, j)\right)$. 
Since $2^{-l_{i}}<\delta$, it follows that $V_{i}^{\prime}=\left\{V^{\prime}(i, 0), \ldots, V^{\prime}\left(i, B_{2 i}\right)\right\}$ is an open taut chain cover of $G_{i} ; V_{i}^{\prime}$ follows $f_{i 1}$ in $g_{i} ; V_{i}^{\prime}$ is an amalgamation of some links of $C_{l_{i}}^{\prime}$; and only end links of $V_{i}^{\prime}$ can contain boundary points of $\bar{O}$.

Define $\hat{C}(2, i, j)=V^{\prime}(i, j), \hat{C}(2, i)=V_{i}^{\prime}$, and $\hat{C}(2)=\left\{V_{1}^{\prime}, \ldots, V_{M_{2}}^{\prime}\right\}$. Note that mesh $\hat{C}(2) \leq 1 / 2$ and $\hat{C}(2)$ follows $f_{1}$ in $\hat{C}(1)$.

There is $k_{3}$ in $N$ such that $C\left(k_{3}\right)$ closure-refines $\hat{C}(2), \hat{C}(2)$ is an amalgamation of $C\left(k_{3}\right)$, and mesh $C\left(k_{3}\right)<\frac{1}{8}$. Define, for appropriate $j, k, \hat{C}(3, j, k)=C\left(k_{3}, j, k\right)$; $\hat{C}(3, j)=C\left(k_{3}, j\right) ; \hat{C}(3)=C\left(k_{3}\right)$. Now obtain $\hat{D}(3)$ in a manner similar to the one in which $\hat{C}(2)$ was just obtained (so that $\hat{D}(3)$ follows a pattern $f_{2}$ in $\hat{D}(2)$ that $\hat{C}(3)$ follows in $\hat{C}(2))$, and continue this process. We obtain the desired sequences, and we use the preceding theorem to conclude that there exists a homeomorphism $h: \bar{O} \rightarrow \bar{U}$.

It remains to show that $h(\partial O)=\partial U$. First we note that $x \in \bar{O}-O$ if and only if $\{x\}=\bigcap c_{i}$ where for each $i, c_{i}$ is an end link of a chain in $\hat{C}(i)$. To see this, recall that $x \in \bar{O}-O$ implies that $x$ is in the closure of exactly one link $C$ of one chain of $\hat{C}(i)$, and that link must be an end link of a chain in $\hat{C}(i)$. On the other hand, if $x$ belongs to an end link of some chain in $\hat{C}^{\prime}(i)$ for each $i$, then $d(x, \bar{O}-O) \leq \operatorname{mesh} \hat{C}(i)$ for all $i$. Hence $x \in \bar{O}-O$.

Similarly $y \in \bar{U}-U$ if and only if $\{y\}=\bigcap d_{i}$ where for each $i, d_{i}$ is an end link of a chain in $\hat{D}(i)$.

Furthermore the patterns $f_{i}$ take end links to end links, and the homeomorphism $h$ takes a point in $\bar{O}$ that is the intersection of end links to a point in $\bar{U}$ that is an intersection of end links. This completes the proof of the theorem.

THEOREM 8. If $A$ is an open set of the pseudoarc $X$ such that $X-\bar{A} \neq \varnothing$, then $A$ is homeomorphic to an open set of the pseudocircle. If $B$ is an open set of the pseudocircle $Y$ such that $Y-\bar{B} \neq \varnothing$, then $B$ is homeomorphic to an open set of the pseudoarc.

ProOF. Without loss of generality, the chain $O_{1}=\left\{O_{1}, \ldots, O_{n}\right\}$ of the previous theorem has the property that neither its first link $O_{1}$ nor its last link $O_{n}$ intersects A.

Let $O=O_{2} \cup \cdots \cup O_{n-1}$. Then $O_{1}^{\prime}=\left\{O_{1}, O, O_{n}\right\}$ is a three-linked chain covering the pseudoarc. Replace $O_{1}$ with $O_{1}^{\prime}$ in the preceding proof. It follows that $A \subset O$ and $O$ is homeomorphic to an open set $U$ of the pseudocircle. Hence $A$ is homeomorphic to an open set of the pseudocircle.

A similar proof holds for the second claim.

5. The main theorem. We now have the ingredients for a proof of the following theorem.

THEOREM 9. The pseudocircle $X$ has uncountably many orbits under the action of its homeomorphism group. Each such orbit is the union of uncountably many composants.

PROOF. If a continuum $Z$ has only countably many orbits under the action of its homeomorphism group, then one of these orbits is second category in $Z$ and hence second category in itself. It follows from the Effros Theorem [4] that $Z$ has a $G_{\delta^{-}}$ orbit. Since the pseudocircle is almost chainable $[\mathbf{2}]$, hereditarily indecomposable, 
and nearly homogeneous, and since each hereditarily indecomposable continuum has property $K[\mathbf{1 4}]$, it follows that the pseudocircle has uncountably many orbits.

Since each orbit of the pseudocircle under the action of the group $G$ of homeomorphisms constructed in the second section meets uncountably many composants, it follows that each orbit of the pseudocircle under the action of $H(X)$ meets uncountably many composants.

Finally, in [10], Lewis proved the following theorem: Let $V$ be an open subset of the pseudoarc $P$. Let $p$ and $q$ be distinct points of $P$ such that the subcontinuum $M$, irreducible between $p$ and $q$, does not intersect $\bar{V}$. Then there exists a homeomorphism $k$ from $P$ onto $P$ with $h(p)=q$ and $k \mid V$ is the identity. Lewis has announced that the techniques of his proof apply as well to the pseudocircle; we use Theorem 7 to prove this directly.

Let $p^{\prime}$ and $q^{\prime}$ be points of the pseudocircle $X$ such that $p^{\prime}$ and $q^{\prime}$ belong to the same composant of $X$. Let $M^{\prime}$ be the subcontinuum irreducible between $p^{\prime}$ and $q^{\prime}$. Let $U=\left\{U_{1}, U_{2}, \ldots, U_{n}\right\}$ be a circular chain covering $X$ such that neither $\bar{U}_{1}$ nor $\bar{U}_{2}$ contains a point of $M^{\prime}$. Let $U=U_{3} \cup \cdots \cup U_{n}$. Then $\left\{U_{1}, U_{2}, U\right\}$ is a three-linked circular chain covering $X$.

Use Theorem 7 to find an open set $O$ of the pseudoarc such that $P-\bar{O} \neq \varnothing$ and a homeomorphism $h$ from $\bar{O}$ to $\bar{U}$ such that $h(\partial \bar{O})=\partial \bar{U}$. Let $p=h^{-1}\left(p^{\prime}\right)$ and $q=h^{-1}\left(q^{\prime}\right)$. Then $M=h^{-1}\left(M^{\prime}\right)$ is an irreducible continuum from $p$ to $q$.

Let $k: P \rightarrow P$ be a homeomorphism such that $k(p)=q$ and $k \mid(P-O)$ is the identity. Then $h \circ k \circ h^{-1}$ is a homeomorphism of the pseudocircle onto itself that takes $p^{\prime}$ onto $q^{\prime}$.

Hence each composant of the pseudocircle is a subset of some orbit of $X$, and the theorem is proved.

\section{REFERENCES}

1. R. H. Bing, Concerning hereditarily indecomposable continua, Pacific J. Math. 1 (1951), 43-51.

2. C. E. Burgess, Homogeneous continua which are almost chainable, Canad. J. Math. 13 (1961), 519-528.

3. Wayne Lewis, Continua theory problems, Topology Proceedings (2) 8 (1983), 361-394.

4. Edward G. Effros, Transformation groups and $C^{*}$-algebras, Ann. of Math. (2) 81 (1965), 38-55.

5. L. Fearnley, The pseudo-circle is not homogeneous, Bull. Amer. Math. Soc. 75 (1969), 554-558.

6. _ The pseudo-circle is unique, Bull. Amer. Math. Soc. 75 (1969), 398-401.

7. Charles L. Hagopian, The fixed point property for almost chainable homogeneous continua, Illinois J. Math. 20 (1976), 650-652.

8. O. H. Hamilton, $A$ fixed point theorem for pseudo-arcs and certain other metric continua, Proc. Amer. Math. Soc. 2 (1951), 173-174.

9. Michael Handel, A pathological area preserving $C^{\infty}$ diffeomorphism of the plane, Proc. Amer. Math. Soc. 86 (1982), 163-168.

10. Wayne Lewis, Almost chainable homogeneous continua are chainable, Houston J. Math. 7 (1981), no. 3, 373-377.

11. _ Stable homeomorphisms of the pseudo-arc, Canad. J. Math. 31 (1979), no. 2, 363374.

12. Lex G. Oversteegen and E. D. Tymchatyn, On hereditarily indecomposable continua, preprint. 
13. James $\mathrm{T}$. Rogers, Jr., Homogeneous separating plane continua are decomposable, Michigan Math. J. 28 (1981), 317-321.

14. __ The pseudo-circle is not homogeneous, Trans. Amer. Math. Soc. 148 (1970), 417428.

15. Roger W. Wardle, On a property of J. L. Kelley, Houston J. Math., 3 (1977), 291-299.

Department of Mathematics, Tulane University, New Orleans, Louisiana 70118

Department of Mathematics, Auburn University, Auburn, Alabama 36849 